\title{
Determinants of plasma dihydrophylloquinone in men and women
}

\author{
Arja T. Erkkilä ${ }^{1,2,3}$, Alice H. Lichtenstein ${ }^{1}$, Paul F. Jacques ${ }^{4}$, Frank B. Hu ${ }^{5}$, Peter W. F. Wilson ${ }^{6}$ \\ and Sarah L. Booth ${ }^{2} *$ \\ ${ }^{1}$ Cardiovascular Nutrition Laboratory and \\ ${ }^{2}$ Vitamin K Laboratory, Jean Mayer USDA Human Nutrition Research Center on Aging at Tufts University, 711 Washington Street, \\ Boston, MA 02111, USA \\ ${ }^{3}$ Department of Clinical Nutrition, University of Kuopio, Kuopio, Finland \\ ${ }^{4}$ Epidemiology Program, Jean Mayer USDA Human Nutrition Research Center on Aging at Tufts University, 711 Washington Street, \\ Boston, MA 02111, USA \\ ${ }^{5}$ Department of Nutrition, Harvard School of Public Health, Boston, MA 02115 and Channing Laboratory, Boston, MA 02115, USA \\ ${ }^{6}$ Medical University of South Carolina, Charleston, SC 29425 and the National Heart, Lung and Blood Institute's Framingham Heart \\ Study, Framingham, MA 01701 USA
}

(Received 28 May 2004 - Revised 3 December 2004 - Accepted 8 December 2004)

\begin{abstract}
Commercial hydrogenation results in the formation of trans fatty acids. An unintended consequence of the hydrogenation process is conversion of phylloquinone (vitamin $\mathrm{K}_{1}$ ) to dihydrophylloquinone. Plasma dihydrophylloquinone concentrations have yet to be characterized in population-based studies. Dietary determinants of plasma dihydrophylloquinone were estimated using a semi-quantitative food frequency questionnaire in 803 men and 913 women in the Framingham Offspring Study. Geometric mean dihydrophylloquinone intake was 21.3 (95\% CI 20.4, 22.3) $\mu \mathrm{g} / \mathrm{d}$ in men and 19.4 (95\% CI $18.5,20 \cdot 2) \mu \mathrm{g} / \mathrm{d}$ in women. Detectable $(>0.05 \mathrm{nmol} / \mathrm{l})$ plasma dihydrophylloquinone concentrations were measured in $41 \%$ and $30 \%$ of men and women, respectively. The multivariate odds ratio (OR) of detectable plasma dihydrophylloquinone from the lowest to the highest quartile category of dihydrophylloquinone intake were 1 (referent), 1.13 (95\% CI 0.83, 1.53), 1.66 (95\% CI 1.21, 2.26) and 1.84 (95\% CI 1.31, 2.58), $P$ for trend <0.001, adjusted for sex, age, body mass index, triacylglycerols, season and energy intake. Higher trans fatty acid intake was associated with higher multivariate OR for detectable plasma dihydrophylloquinone (OR comparing extreme quartiles 2.41 (95\% CI 1.59, 3.64), P for trend <0.001). There were limitations in the use of plasma dihydrophylloquinone, evident in the high proportion of the population that had non-detectable dihydrophylloquinone concentrations. Despite this caveat, higher plasma dihydrophylloquinone was associated with higher dihydrophylloquinone intake and higher trans fatty acid intake.
\end{abstract}

Dihydrophylloquinone: Vitamin K: Trans fatty acids: Biomarker

\section{Introduction}

Plant oils are hydrogenated to alter the physical characteristics of the fat and increase chemical stability (Federal Register, 2003). This process increases the potential applications of the fat for commercial uses; hence the practice is widespread in many countries. The oils most commonly hydrogenated in the USA are soyabean and rapeseed, which are also rich in dietary sources of phylloquinone (also known as vitamin $\mathrm{K}_{1}$; Booth \& Suttie, 1998). An unintended consequence of the hydrogenation process is the saturation of the $2^{\prime}, 3^{\prime}$ double bond on the side chain of phylloquinone, resulting in the formation of $2^{\prime}, 3^{\prime}$-dihydrophylloquinone (Davidson et al. 1996). This change is of particular interest in light of prior work suggesting that while dihydrophylloquinone is absorbed from hydrogenated plant oils (Booth et al. $1996 a$, 2001), the biological activity of dihydrophylloquinone is less compared with its parent compound, phylloquinone (Booth et al. 2001).

Since 1990, there has been considerable interest in dietary trans fatty acid intakes because of potential adverse physiological consequence(s) associated with this class of fatty acids on plasma lipid and lipoprotein concentrations (Mensink \& Katan, 1990; Lichtenstein et al. 1999). Estimation of dietary trans fatty acid intakes from self-reported data, especially from food frequency questionnaires (FFQ), has been hampered by uncertainty in accurately identifying the type of fats used for preparation of specific foods, especially commercially baked and fried foods or mixed dishes, and the completeness of the database for the trans fatty acid content of foods (Federal Register, 2003). For this reason, it would be useful to have a biological marker of trans fatty acid intake that was independent of the aforementioned

Abbreviations: FFQ, food frequency questionnaire; OR, odds ratio; ucOC, undercarboxylated osteocalcin.

Disclaimer: This material is based upon work supported by the US Department of Agriculture, under agreement No. 58-1950-4-401. Any opinions, findings, conclusions or recommendations expressed in this publication are those of the authors and do not necessarily reflect the view of the US Department of Agriculture.

* Corresponding author: Dr Sarah L. Booth, fax +1 617556 3149, email sarah.booth@tufts.edu 
limitations. Because the sole source of dietary dihydrophylloquinone is from hydrogenated fat, and the majority of trans fatty acids comes from hydrogenated fat, plasma dihydrophylloquinone may serve as a surrogate marker for trans fatty acid intakes.

The first aim of this study was to investigate the feasibility of quantitating plasma dihydrophylloquinone in the Framingham Offspring Study (Kannel et al. 1979), and examining the associations between this biological marker and estimated dietary dihydrophylloquinone intakes. The second aim of this study was to assess the association between estimated trans fatty acid intake and plasma dihydrophylloquinone concentrations. The final aim of this study was to examine associations between plasma dihydrophylloquinone and biological markers of vitamin $\mathrm{K}$ status.

\section{Methods}

\section{Study population}

The Framingham Offspring Study is a longitudinal, communitybased study of cardiovascular disease among the children and their spouses of the participants in the original Framingham Heart Study cohort (Kannel et al. 1979). The Offspring cohort (original $n$ 5135) has undergone repeat examinations every 3 or 4 years since 1971. Data collection was performed between 1996 and 2000, during the sixth and seventh examination cycles of the Framingham Offspring Study. Subjects taking oral anticoagulants were excluded from the analyses $(n$ 35). Valid FFQ data, covariate information and corresponding blood samples for phylloquinone and dihydrophylloquinone measurements were obtained from 1716 subjects. This study was approved by the Institutional Review Boards of Tufts-New England Medical Center and Boston University.

\section{Dietary assessment}

Usual dietary intake during the previous year was assessed using a semi-quantitative FFQ, as previously described (Willett et al. 1985; Rimm et al. 1992). The questionnaires were mailed to the participants before the examination, and completed questionnaires were returned at the examination. Dietary data were excluded if the subjects reported energy intake $<2.51 \mathrm{MJ} / \mathrm{d}$ $(600 \mathrm{kcal} / \mathrm{d})$ or $>16.74 \mathrm{MJ} / \mathrm{d} \quad(4000 \mathrm{kcal} / \mathrm{d})$ for women and $>17.57 \mathrm{MJ} / \mathrm{d}(4200 \mathrm{kcal} / \mathrm{d})$ for men, or $\geq 12$ food items were left blank. Daily dietary dihydrophylloquinone intake was calculated by multiplying the dihydrophylloquinone content per serving of each food (Booth et al. 1996b; Peterson et al. 2002; Dumont et al. 2003) by the reported frequency of consumption, and summing over all foods. The dihydrophylloquinone content of foods was based on recent laboratory analysis of geographically representative foods collected as part of the US Department of Agriculture's National Food and Nutrient Analysis Plan, as described elsewhere (Peterson et al. 2002). The FFQ included questions on the frequency of consumption of fried food consumed either out or in the home. The number of servings of bakery products was computed by summing the servings of crackers, cookies, brownies, doughnuts, cake, sweet rolls and pies. Servings of hot dogs, chicken/turkey dogs, French fries, pizza and tortilla were included in the category of fast food and servings of popcorn, potato chips and corn chips in the category of snacks. Data on trans fatty acid intake were available for 1238 of the 1716 subjects used in these analyses.

\section{Blood measurements}

Plasma phylloquinone and dihydrophylloquinone concentrations were determined by reverse-phase HPLC with use of postcolumn reduction and fluorometric detection (Davidson \& Sadowski, 1997). The lower limit of detection for plasma phylloquinone and dihydrophylloquinone was $0.05 \mathrm{nmol} / 1$. Serum total osteocalcin and undercarboxylated osteocalcin were measured by RIA (Gundberg et al. 1998). Undercarboxylated osteocalcin was expressed as the percentage of osteocalcin not bound to hydroxyapatite in vitro (\%ucOC), and normalized to the amount of total osteocalcin in a given sample. Plasma triacylglycerols were measured using standardized enzymatic methods (McNamara \& Schaefer, 1987).

\section{Covariate measurements}

Anthropometric measurements were taken at the time of the examination and BMI $\left(\mathrm{kg} / \mathrm{m}^{2}\right)$ was calculated. Subjects were classified as abdominal obese if waist circumference was $>88 \mathrm{~cm}$ in females and $>102 \mathrm{~cm}$ in males (National Cholesterol Education Program Expert Panel, 2002). Data on regular smoking during the previous year (yes/no) $(\mathrm{Y} / \mathrm{N})$, menopausal status (cessation of menses for at least 1 year) and current use of hormone replacement therapy $(\mathrm{Y} / \mathrm{N})$ were collected at the time of the examination. Season (winter, spring, summer, autumn) was also used as covariate.

\section{Statistical methods}

All statistical analyses were performed using Statistical Analysis System, version 8.2 (SAS Institute, Cary, NC, USA). To improve normality for statistical testing, natural logarithmic transformations were applied to dietary dihydrophylloquinone intake and plasma phylloquinone. Adjusted geometric means and $95 \% \mathrm{CI}$ were calculated for these variables. Because plasma dihydrophylloquinone was non-detectable (defined as $\leq 0.05 \mathrm{nmol} / \mathrm{l}$ ) in $64 \%$ of the samples and the distribution was skewed toward lower values, the percentage of detectable (defined as $>0.05 \mathrm{nmol} / \mathrm{l}$ ) concentrations, with $95 \% \mathrm{CI}$, are presented, instead of actual concentrations. Because distribution of \%ucOC was skewed toward lower values, the data are presented as percentage of subjects having $\geq 20 \%$ ucOC, with $95 \% \mathrm{CI}$, which is suggested to reflect low vitamin $\mathrm{K}$ intake, based on metabolic data using the same assay (Booth et al. 1999a, 2001).

Possible determinants of dietary intake and plasma concentrations $>0.05 \mathrm{nmol} / 1$ of dihydrophylloquinone included sex, age $(<50,50-59,60-69, \geq 70$ years), BMI $(<25,25-30$, $\left.\geq 30 \mathrm{~kg} / \mathrm{m}^{2}\right)$, abdominal adiposity $(>88 \mathrm{~cm}$ for women, $>102 \mathrm{~cm}$ for men), season (winter, spring, summer, autumn), postmenopausal status $(\mathrm{Y} / \mathrm{N})$ and current hormone replacement therapy $(\mathrm{Y} / \mathrm{N})$. Differences in geometric mean dihydrophylloquinone intake and proportion of detectable plasma dihydrophylloquinone across categories of these potential determinants were assessed using SAS PROC GLM and Scheffé's test for pairwise comparisons. In addition, geometric mean dihydrophylloquinone intake levels were adjusted for energy intake and the proportion of detectable plasma dihydrophylloquinone concentrations was presented with and without adjustment for plasma triacylglycerol concentrations. Analyses of plasma dihydrophylloquinone were adjusted for plasma triacylglycerols in the model because 
previously metabolic studies suggest that the triacylglycerol-rich lipoprotein fraction is the main carrier of vitamin $\mathrm{K}$ in circulation (Lamon-Fava et al. 1998; Erkkilä et al. 2004). SAS PROC GLM was also used to examine the relation between quartile categories of dihydrophylloquinone intake and detectable levels of plasma dihydrophylloquinone and indicators of vitamin $\mathrm{K}$ status, including plasma phylloquinone and percentage of subjects with elevated \%ucOC. In these analyses, geometric mean plasma phylloquinone concentrations and proportions of detectable plasma dihydrophylloquinone concentrations were adjusted for plasma triacylglycerol concentrations, and dihydrophylloquinone intakes were adjusted for energy intake.

Multivariate logistic regression analyses were used to calculate odds ratios (OR), with $95 \% \mathrm{CI}$, for detectable plasma dihydrophylloquinone concentrations (defined as $>0.05 \mathrm{nmol} / \mathrm{l}$ ) across quartile categories of dietary dihydrophylloquinone, trans fatty acids or foods rich in dihydrophylloquinone adjusting for significant correlates of either dihydrophylloquinone intake or plasma dihydrophylloquinone as well as energy intake and tertile categories of plasma triacylglycerols. Tests of linear trend across increasing categories of dietary dihydrophylloquinone, trans fatty acids or foods rich in dihydrophylloquinone were conducted by assigning the medians of intakes to categories that were treated as a continuous variable. Spearman correlation coefficients were calculated between plasma dihydrophylloquinone and triacylglycerols and between dihydrophylloquinone and trans fatty acid intakes. A value of $P<0 \cdot 05$ (two-tailed) was considered statistically significant.

\section{Results}

The mean plasma dihydrophylloquinone concentrations for all subjects were 0.31 (SD 0.76) and 0.19 (SD 0.41) nmol/l for men and women, respectively. The percentage of those with detectable plasma dihydrophylloquinone was higher in men than in women (41\% v. $31 \%, P<0.001$; Table 1). There was a positive correlation between plasma dihydrophylloquinone and triacylglycerol concentrations $(r 0.21, P<0.001)$, so data are presented as adjusted and unadjusted for triacylglycerols in Table 1 . Subjects with $\mathrm{BMI}>30 \mathrm{~kg} / \mathrm{m}^{2}$ or abdominal obesity were more likely to have detectable plasma dihydrophylloquinone. However, these associations became non-significant after adjustment for triacylglycerol concentrations. Among postmenopausal women, those who were taking hormone replacement therapy had less detectable plasma dihydrophylloquinone concentrations than those who were not. The percentage of subjects with detectable plasma dihydrophylloquinone was higher in summer than in spring.

Table 1. Mean percentage of subjects with detectable plasma dihydrophylloquinone concentrations $(>0.05 \mathrm{nmol} / \mathrm{l})$ by categories of potential determinants (Mean values with their $95 \% \mathrm{Cl}$ )

\begin{tabular}{|c|c|c|c|c|c|c|c|}
\hline & $n$ & \multicolumn{2}{|c|}{$\begin{array}{c}\% \text { Detectable plasma } \\
\text { dihydrophylloquinone, } \\
\text { unadjusted for triacylglycerols }\end{array}$} & $P^{\star}$ & \multicolumn{2}{|c|}{$\begin{array}{c}\text { \% Detectable plasma } \\
\text { dihydrophylloquinone, } \\
\text { adjusted for triacylglycerols }\end{array}$} & $P^{\star}$ \\
\hline \multicolumn{8}{|l|}{ Sex } \\
\hline Men & 803 & $41 \cdot 2$ & $38 \cdot 0,44 \cdot 5$ & & $40 \cdot 7$ & $37 \cdot 5,44 \cdot 0$ & \\
\hline Women & 913 & 30.6 & $27 \cdot 5,33 \cdot 6$ & $<0.001$ & $31 \cdot 0$ & $27 \cdot 9,34 \cdot 0$ & $<0.001$ \\
\hline$<50$ & 290 & $32 \cdot 1$ & $26 \cdot 6,37 \cdot 6$ & & 34.0 & $28 \cdot 6,39 \cdot 4$ & \\
\hline $50-59$ & 690 & $33 \cdot 9$ & $30 \cdot 3,37 \cdot 5$ & & 33.6 & $30 \cdot 1,37 \cdot 1$ & \\
\hline $60-69$ & 496 & $36 \cdot 9$ & $32 \cdot 7,41 \cdot 1$ & & $36 \cdot 3$ & $32 \cdot 1,40 \cdot 4$ & \\
\hline$\geq 70$ & 240 & $41 \cdot 7$ & $35 \cdot 6,47 \cdot 7$ & 0.09 & $41 \cdot 5$ & $35.5,47.5$ & 0.15 \\
\hline \multicolumn{8}{|l|}{ BMI $\left(\mathrm{kg} / \mathrm{m}^{2}\right)$} \\
\hline$<25$ & 512 & $29 \cdot 9^{a}$ & $25 \cdot 7,34 \cdot 0$ & & $32 \cdot 2$ & $28 \cdot 0,36 \cdot 3$ & \\
\hline $25-30$ & 703 & $35 \cdot 7^{\mathrm{ab}}$ & $32 \cdot 2,39 \cdot 2$ & & $35 \cdot 9$ & $32 \cdot 4,39 \cdot 4$ & \\
\hline$>30$ & 499 & $41 \cdot 3^{b}$ & $37 \cdot 1,45 \cdot 5$ & $<0.001$ & $38 \cdot 7$ & $34 \cdot 4,42 \cdot 9$ & 0.11 \\
\hline No & 815 & $32 \cdot 8$ & $29 \cdot 5,36 \cdot 0$ & & 34.4 & $31 \cdot 1,37 \cdot 7$ & \\
\hline Yes & 901 & $38 \cdot 1$ & $34 \cdot 9,41 \cdot 2$ & 0.03 & $36 \cdot 6$ & $33.5,39.7$ & 0.35 \\
\hline \multicolumn{8}{|l|}{ Current smoker } \\
\hline No & 1464 & $35 \cdot 5$ & $33 \cdot 1,38 \cdot 0$ & & $35 \cdot 6$ & $33 \cdot 2,38 \cdot 0$ & \\
\hline Yes & 252 & $35 \cdot 7$ & $29 \cdot 8,41 \cdot 6$ & 0.96 & $35 \cdot 3$ & $29 \cdot 5,41 \cdot 2$ & 0.95 \\
\hline \multicolumn{8}{|l|}{ Season } \\
\hline Winter (Dec-Feb) & 526 & $37 \cdot 1^{\mathrm{ab}}$ & $33 \cdot 0,41 \cdot 2$ & & $37.7^{\mathrm{ab}}$ & $33 \cdot 6,41 \cdot 7$ & \\
\hline Spring (Mar-May) & 506 & $30 \cdot 4^{\mathrm{a}}$ & $26 \cdot 3,34 \cdot 6$ & & $29 \cdot 9^{a}$ & $25 \cdot 8,34 \cdot 0$ & \\
\hline Summer (June-Aug) & 187 & $42 \cdot 8^{b}$ & $35 \cdot 9,49 \cdot 6$ & & $42 \cdot 6^{\mathrm{b}}$ & $35 \cdot 9,49 \cdot 4$ & \\
\hline Fall (Sep-Nov) & 497 & $36 \cdot 4^{\mathrm{ab}}$ & $32 \cdot 2,40 \cdot 6$ & 0.02 & $36 \cdot 4^{\mathrm{ab}}$ & $32 \cdot 3,40 \cdot 6$ & 0.006 \\
\hline \multicolumn{8}{|c|}{ Postmenopausal status, women } \\
\hline No & 215 & $24 \cdot 7$ & $18 \cdot 5,30 \cdot 8$ & & $26 \cdot 8$ & $20 \cdot 6,33 \cdot 0$ & \\
\hline Yes & 698 & $32 \cdot 4$ & $29 \cdot 0,35 \cdot 8$ & 0.04 & $31 \cdot 7$ & $28 \cdot 3,35 \cdot 1$ & $0 \cdot 18$ \\
\hline \multicolumn{8}{|c|}{ Hormone replacement therapy, postmenopausal women } \\
\hline No & 439 & $32 \cdot 2$ & $28 \cdot 6,35 \cdot 8$ & & $32 \cdot 7$ & $29 \cdot 1,36 \cdot 3$ & \\
\hline Yes & 259 & $27 \cdot 2$ & $21.9,32.5$ & 0.13 & $26 \cdot 1$ & $20 \cdot 9,31.4$ & 0.05 \\
\hline
\end{tabular}

a,b Mean values without common letters are significantly different at $P<0.05$ (Scheffé's test).

* Assessed using SAS PROC GLM.

$\dagger$ Abdominal obesity: waist $>88 \mathrm{~cm}$ in women, $>102 \mathrm{~cm}$ in men. 
Geometric mean intakes of dihydrophylloquinone, adjusted for energy intake, were higher in men $(P=0.004)$, older adults $(P<0.001)$, those who had a BMI $>30 \mathrm{~kg} / \mathrm{m}^{2}(P=0.02)$ and subjects with abdominal obesity $(P=0 \cdot 04$; Table 2$)$. Among postmenopausal women, those who were taking hormone replacement therapy had lower intakes of dihydrophylloquinone than those who were not $(P=0.02)$. The major sources of dihydrophylloquinone for the study cohort are listed in Table 3. Bakery products contributed the majority $(42.4 \%$ and $41.3 \%$ for men and women, respectively) of dihydrophylloquinone intake. Fast foods contributed $28.9 \%$ and $23.6 \%$ of the dihydrophylloquinone intake in men and women, respectively. The correlation between dietary dihydrophylloquinone and trans fatty acid intake was $r 0.78, P<0.001$.

Multivariate OR for detectable plasma dihydrophylloquinone concentrations adjusted for sex, age, BMI, triacylglycerol, season and energy intake was $1.84(95 \%$ CI $1.31,2.58)$ in the highest quartile category of dihydrophylloquinone intake as compared to the lowest quartile ( $P$ for trend $<0.001$; Fig. 1 ). When analysed according to gender, the association was significant both in men and women (OR in increasing quartile categories 1 (referent), 1.37 (95\% CI 0.89, 2.09), 1.76 (95\% CI 1.13, 2.73) and 2.29 (95\% CI $1.41,3.72)$, respectively, in men ( $P$ for trend

Table 2. Geometric mean dihydrophylloquinone intake* by categories of potential determinants (Geometric mean values, adjusted for energy intake, with their $95 \% \mathrm{Cl}$ )

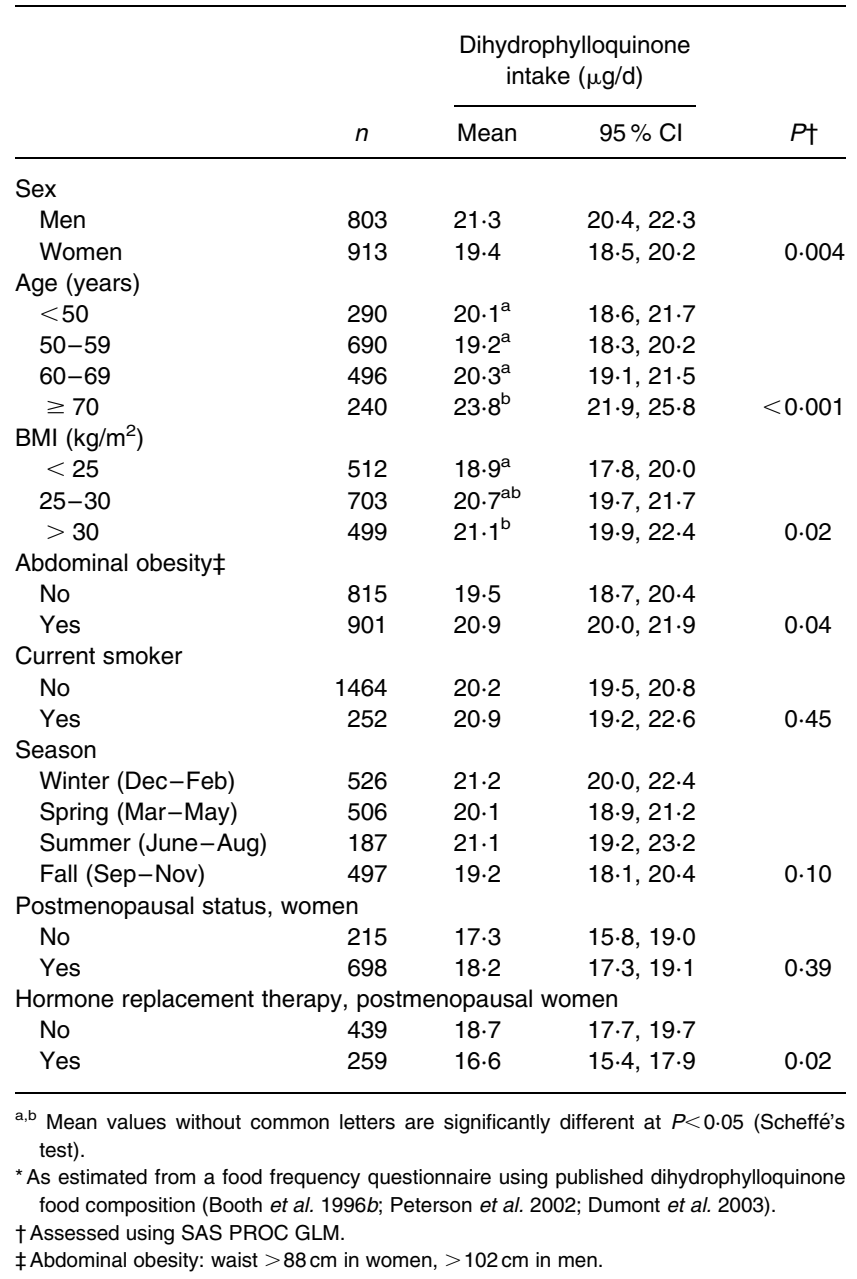

Table 3. Mean contribution of different food items to total dihydrophylloquinone intake*

\begin{tabular}{|c|c|c|}
\hline \multirow[b]{2}{*}{ Food } & \multicolumn{2}{|c|}{ Mean contribution (\%) } \\
\hline & Men ( $n$ 803) & Women ( $n$ 913) \\
\hline Total bakery products $\dagger$ & $42 \cdot 4$ & $41 \cdot 3$ \\
\hline Cookies & $19 \cdot 7$ & $20 \cdot 1$ \\
\hline Sweet rolls & $7 \cdot 2$ & $7 \cdot 7$ \\
\hline Pies & $6 \cdot 7$ & 5.5 \\
\hline Doughnuts & $4 \cdot 2$ & $2 \cdot 7$ \\
\hline Total fast food $\ddagger$ & $28 \cdot 9$ & $23 \cdot 6$ \\
\hline French fries & $16 \cdot 1$ & $11 \cdot 7$ \\
\hline Pizza & $12 \cdot 0$ & $11 \cdot 4$ \\
\hline Snacks§ & $4 \cdot 0$ & $5 \cdot 1$ \\
\hline \multicolumn{3}{|l|}{ Other foods } \\
\hline Mayonnaise & 4.9 & $7 \cdot 1$ \\
\hline Margarine & $4 \cdot 6$ & $5 \cdot 1$ \\
\hline Coffee whitener & $4 \cdot 6$ & $5 \cdot 1$ \\
\hline
\end{tabular}

* As estimated from a food frequency questionnaire using published dihydrophylloquinone food composition (Booth et al. 1996b; Peterson et al. 2002; Dumont et al. 2003). Other food items contributed $<2 \%$ to total dihydrophylloquinone intake.

†Bakery products include crackers, cookies, brownies, doughnuts, cake, sweet rolls and pies.

$\ddagger$ Fast food includes hot dogs, chicken/turkey dogs, French fries, pizza and tortilla. $\S$ Snacks include popcorn, potato and corn chips.

$0.002)$; and 1 (referent), 1.15 (95\% CI 0.74, 1.79), 1.75 (95\% CI $1.12,2.74)$ and $1.82(95 \%$ CI $1.13,2.96)$, respectively, in women $(P$ for trend 0.02$)$ ).

Compared with the lowest intake category of trans fatty acids, the odds of having a detectable concentration of plasma dihydrophylloquinone in the highest quartile category was three times higher in men and almost two times higher in women (Table 4). Higher intake of bakery products was associated with higher OR for detectable plasma dihydrophylloquinone in the whole sample $(P=0.007)$ and in men $(P=0.03)$; in women, the association did not reach significance $(P=0 \cdot 11)$. There was a marginal trend $(P=0.13)$ for a higher OR for detectable plasma dihydrophylloquinone among those subjects who reported eating fried food out, but not fried food prepared at home, at least once a week.

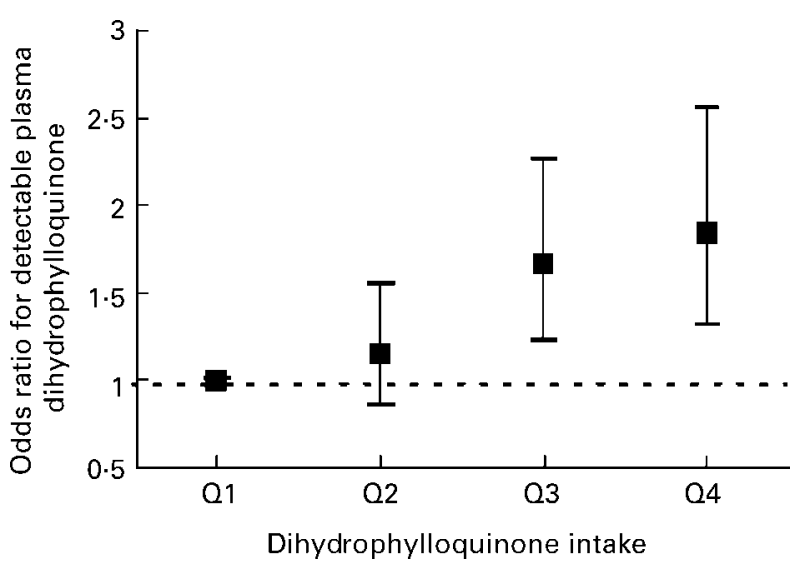

Fig. 1. Multivariate odds ratios (with $95 \% \mathrm{Cl}$ ) for detectable plasma dihydrophylloquinone concentrations $(>0.05 \mathrm{nmol} / \mathrm{l})$ according to median intake in quartile categories (Q1-Q4) of dihydrophylloquinone $(8.2,16.4,26.7$ and $49.1 \mu \mathrm{g} / \mathrm{d}$, respectively) in $427,432,429$ and 428 subjects, respectively, $P<0.001$. The odds ratios are adjusted for sex, age (four groups), BMl (three groups), plasma triacylglycerols (tertiles), season and energy intake (continuous). 
Determinants of plasma dihydrophylloquinone

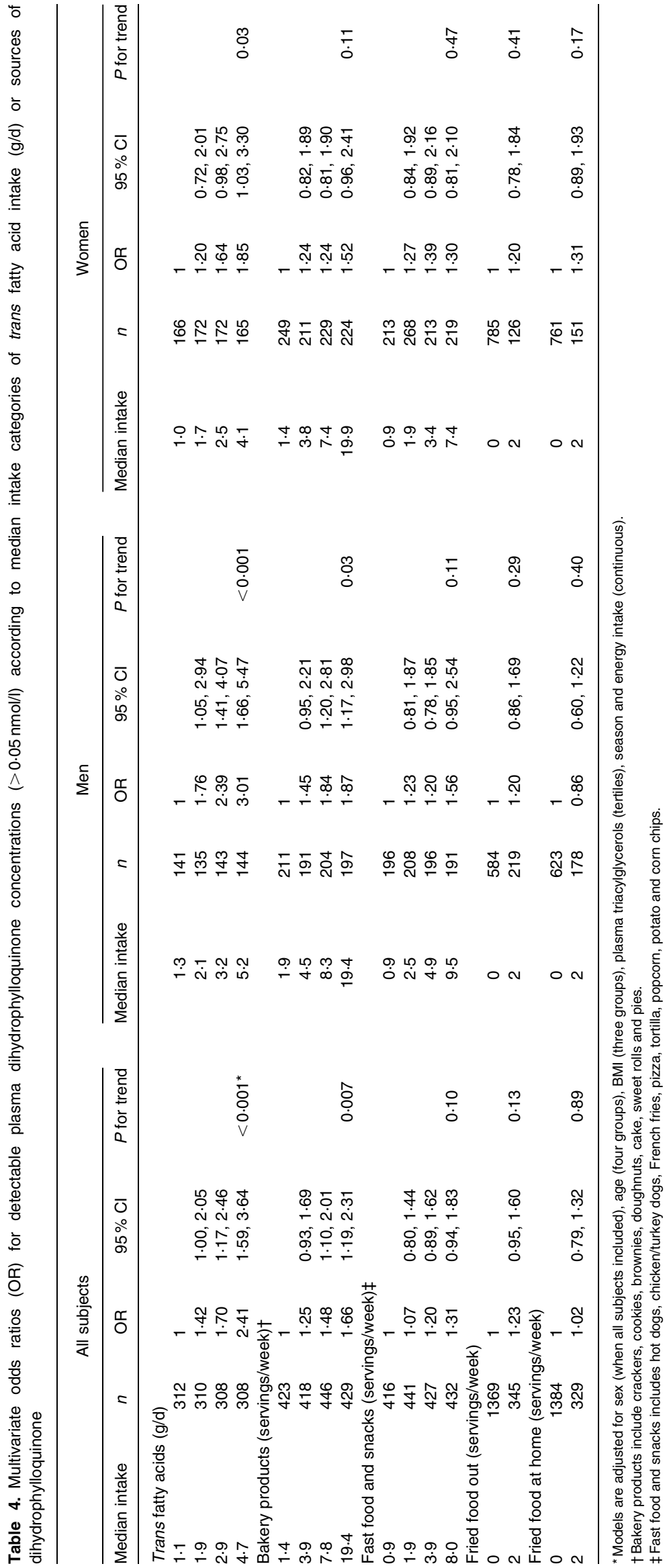


Geometric mean plasma phylloquinone concentrations were higher $(P<0 \cdot 001)$ in those with detectable plasma dihydrophylloquinone (Table 5). The plasma phylloquinone-plasma dihydrophylloquinone association did not change when plasma triacylglycerols were removed from the model (data not shown). However, plasma phylloquinone concentrations were not associated with dihydrophylloquinone intake (Table 6). As a group, those who had higher dihydrophylloquinone intakes tended to have a higher percentage of elevated \%ucOc $(P=0.07$; Table 6$)$. There was no significant sex interaction; however, the association between dihydrophylloquinone intake and percentage of elevated \%ucOc was significant only in women. Somewhat surprisingly, the proportion of elevated \%ucOC did not differ between those subjects who had non-detectable or detectable plasma dihydrophylloquinone (Table 5).

\section{Discussion}

To the best of our knowledge, this is the first report of a positive association between plasma dihydrophylloquinone concentrations and dihydrophylloquinone intake, as assessed with an FFQ, in a free-living population. A positive association was likewise observed between trans fatty acid intakes and detectable plasma dihydrophylloquinone concentrations.

There was a wide range of dihydrophylloquinone intakes in both the current study using the FFQ $(0-140 \mu \mathrm{g}$ dihydrophylloquinone/d), and in a previous study using the $14 \mathrm{~d}$ food diaries (range: 0-220 $\mu \mathrm{g}$ dihydrophylloquinone/d; Booth et al. 1999b). The geometric mean intakes of dihydrophylloquinone in men and women in the current study were in the same range as reported previously in 60-65-year-old men and women using $3 \mathrm{~d}$ dietary intake data (19 and $17 \mu \mathrm{g} / \mathrm{d}$, respectively; Booth et al. 1996b), and in adult men and women using $14 \mathrm{~d}$ food diaries (19 and $15 \mu \mathrm{g} / \mathrm{d}$, respectively; Booth et al. 1999b).

The large proportion of individuals with non-detectable concentrations of plasma dihydrophylloquinone was a limitation

Table 5. Concentrations of plasma phylloquinone and percentage of subjects with elevated ( $\geq 20 \%$ ) undercarboxylated osteocalcin (ucOC) according to categories of plasma dihydrophylloquinone concentrations (Mean values with their $95 \% \mathrm{Cl}$ )

\begin{tabular}{|c|c|c|c|c|c|}
\hline & \multicolumn{4}{|c|}{ Plasma dihydrophylloquinone $(\mathrm{nmol} / \mathrm{l})^{*}$} & \multirow[b]{3}{*}{$P \dagger$} \\
\hline & \multicolumn{2}{|c|}{$\leq 0.05$} & \multicolumn{2}{|c|}{$>0.05$} & \\
\hline & Mean & $95 \% \mathrm{Cl}$ & Mean & $95 \% \mathrm{Cl}$ & \\
\hline \multicolumn{6}{|c|}{ Plasma phylloquinone (nmol/l) } \\
\hline All & $0.86 \ddagger$ & $0.82,0.91$ & $1 \cdot 14$ & $1 \cdot 06,1 \cdot 22$ & $<0.001$ \\
\hline Men & 0.89 & $0.82,0.97$ & $1 \cdot 16$ & $1 \cdot 06,1 \cdot 27$ & $<0.001$ \\
\hline Women & 0.84 & $0.79,0.90$ & $1 \cdot 12$ & $1 \cdot 02,1 \cdot 24$ & $<0.001$ \\
\hline \multicolumn{6}{|l|}{ ucOC (\%) } \\
\hline All & $43.5 \S$ & $40 \cdot 6,46 \cdot 5$ & $43 \cdot 8$ & $39 \cdot 8,47 \cdot 9$ & 0.91 \\
\hline Men & $40 \cdot 2$ & $35 \cdot 7,44 \cdot 7$ & $40 \cdot 4$ & $35 \cdot 0,45 \cdot 8$ & 0.95 \\
\hline Women & $46 \cdot 2$ & $42 \cdot 2,50 \cdot 1$ & $47 \cdot 8$ & $41 \cdot 8,53 \cdot 7$ & 0.66 \\
\hline
\end{tabular}

* In $\leq 0.05$ and $>0.05 \mathrm{nmol} / \mathrm{l}$ categories, number of all subjects was 1106 and 610 , respectively, for plasma phylloquinone, and 1087 and 599, respectively, for ucOC; number of men was 472 and 331 for plasma phylloquinone, and 467 and 323, respectively, for ucOC; number of women was 634 and 279 , respectively, for plasma phylloquinone, and 620 and 276 , respectively, for ucOC.

†SAS PROC GLM

† Geometric means adjusted for plasma triacylglycerol.

§Proportion of high $(\geq 20 \%)$ ucOC adjusted for plasma triacylglycerol. of the current study. Absolute reported intakes of dihydrophylloquinone were lower compared with those of phylloquinone previously reported for the Framingham Offspring Cohort (McKeown et al. 2002) so it is not unexpected that corresponding plasma dihydrophylloquinone concentrations were low. Dihydrophylloquinone may also be absorbed to a lesser extent or more rapidly metabolized and excreted compared with an equivalent amount of phylloquinone (Booth et al. 2001). In one metabolic study (Booth et al. 1996a), the plasma dihydrophylloquinone concentrations were all within detectable range (mean dihydrophylloquinone concentration of $0.56 \mathrm{nmol} / \mathrm{l}$ ) in response to daily intakes of $23 \mu \mathrm{g}$ dihydrophylloquinone. However, this study (Booth et al. 1996a) was limited to eight mildly hyperlipidaemic men and women, and it is plausible that the higher plasma dihydrophylloquinone concentrations were associated with higher triacylglycerol concentrations. It is assumed that dihydrophylloquinone would have a similar transport mechanism as the parent form phylloquinone, which is carried primarily by triacylglycerol-rich lipoproteins (Lamon-Fava et al. 1998; Erkkilä et al. 2004). Although there is a positive correlation between plasma phylloquinone and plasma triacylglycerols (McKeown et al. 2002), there is current controversy regarding the necessity to adjust plasma phylloquinone for plasma lipids (Traber \& Jialal, 2000). Even less is known about correction of plasma dihydrophylloquinone for plasma lipids. Regardless of the mechanism(s), low plasma dihydrophylloquinone concentrations present analytical challenges when assessed in a free-living population that has a wide range of lipid concentrations and dihydrophylloquinone intakes.

Higher BMI and abdominal obesity were associated with higher dihydrophylloquinone intake, even after adjustment for energy intake. Whereas plasma dihydrophylloquinone was also positively associated with measures of obesity, further adjustment for plasma triacylglycerols resulted in a loss of statistical significance. It is not known whether the attenuation of associations with plasma dihydrophylloquinone following adjustment for plasma triacylglycerols were due to positive correlations between measures of obesity and plasma triacylglycerols (Grundy, 1998).

The hydrogenation process results in the formation of both trans fatty acids and dihydrophylloquinone (Davidson et al. 1996). The dihydrophylloquinone content in margarines has been reported to correlate with that of trans fatty acids (Koivu et al. 1999). However, sources of dihydrophylloquinone and trans fatty acids are not entirely the same. All dihydrophylloquinone comes from foods made with hydrogenated vegetable oils and is not naturally occurring. Whereas the majority of trans fatty acids $(80 \%)$ comes from foods made with hydrogenated vegetable oil, $20 \%$ comes from endogenous production in ruminant animals which accumulates in the fat (Federal Register, 2003). Using data from $3 \mathrm{~d}$ food intake diaries collected as part of the Continuing Survey of Food Intakes by Individuals (CSFII 94-96; Federal Register, 2003), the major foods that contribute to trans fatty acid intake from hydrogenated fat include bakery products $(42 \%$ of the total trans fatty acid intake from products made with hydrogenated fat), margarine $(21 \%)$, French fries $(11 \%)$, potato and corn chips $(6 \%)$ and shortening $(5 \%)$. These contributions are similar to that observed for dihydrophylloquinone in this cohort, with the exception of margarine, which contributed less $(5 \%)$.

Having similar dietary sources, it was not unexpected that a positive association was observed between dihydrophylloquinone 
Table 6. Concentrations of plasma phylloquinone and percentage of subjects with elevated ( $\geq 20 \%$ ) undercarboxylated osteocalcin (ucOC) according to quartile categories $(Q)$ of dihydrophylloquinone intake ${ }^{*}$

(Mean values with their $95 \% \mathrm{Cl}$ )

\begin{tabular}{|c|c|c|c|c|c|c|c|c|c|}
\hline & \multicolumn{8}{|c|}{ Dihydrophylloquinone intake $†$} & \multirow[b]{3}{*}{$P \ddagger$} \\
\hline & \multicolumn{2}{|c|}{ Q1 (low) } & \multicolumn{2}{|c|}{ Q2 } & \multicolumn{2}{|c|}{ Q3 } & \multicolumn{2}{|c|}{ Q4 (high) } & \\
\hline & Mean & $95 \% \mathrm{Cl}$ & Mean & $95 \% \mathrm{Cl}$ & Mean & $95 \% \mathrm{Cl}$ & Mean & $95 \% \mathrm{Cl}$ & \\
\hline \multicolumn{10}{|c|}{ Plasma phylloquinone (nmol/l) } \\
\hline All & $0.98 \S$ & $0.90,1.07$ & 0.97 & $0.89,1.05$ & 0.97 & $0.89,1.06$ & 0.89 & $0.82,0.98$ & 0.48 \\
\hline Men & $1 \cdot 11$ & $0.98,1.27$ & 0.96 & $0.85,1.09$ & 0.96 & $0.85,1.08$ & 0.95 & $0.83,1.09$ & 0.28 \\
\hline Women & 0.90 & $0.80,1.01$ & 0.91 & $0.81,1.01$ & 1.03 & $0.92,1.15$ & 0.86 & $0.76,0.96$ & 0.13 \\
\hline \multicolumn{10}{|l|}{ ucOC (\%) } \\
\hline All & $38 \cdot 0 \|$ & $32 \cdot 9,43 \cdot 1$ & $44 \cdot 5$ & $39 \cdot 8,49 \cdot 3$ & $43 \cdot 6$ & $38 \cdot 9,48 \cdot 4$ & $48 \cdot 4$ & $43 \cdot 3,53 \cdot 6$ & 0.07 \\
\hline Men & $36 \cdot 4$ & $29 \cdot 1,43 \cdot 6$ & $38 \cdot 7$ & $31 \cdot 7,45 \cdot 6$ & $41 \cdot 1$ & $34 \cdot 2,47 \cdot 9$ & $45 \cdot 0$ & $37 \cdot 4,52 \cdot 6$ & 0.49 \\
\hline Women & $38 \cdot 6^{a}$ & $31.5,45 \cdot 6$ & $49 \cdot 2^{\mathrm{ab}}$ & $42 \cdot 7,55 \cdot 8$ & $44 \cdot 5^{\mathrm{ab}}$ & $37 \cdot 9,51 \cdot 1$ & $54 \cdot 3^{b}$ & $47 \cdot 2,61 \cdot 3$ & 0.02 \\
\hline
\end{tabular}

a,b Mean values without common letters are significantly different at $P<0.05$ (Scheffé's test).

* As estimated from a food frequency questionnaire using published dihydrophylloquinone food composition (Booth et al. 1996b; Peterson et al. 2002; Dumont et al. 2003).

† Median intakes in the quartile categories were 8.2, 16.4, $26 \cdot 7$ and $49.1 \mu \mathrm{g} / \mathrm{d}$, respectively, in all subjects; $10 \cdot 2,19.4,29.1 \mathrm{and} 54.4 \mu \mathrm{g} / \mathrm{d}$, respectively, in men; and 7.2, $14.1,23.9$ and $46.3 \mu \mathrm{g} / \mathrm{d}$, respectively, in women. In quartile categories, number of all subjects was $427,432,429$ and 428 , respectively, for plasma phylloquinone, and 419, 426, 420 and 421, respectively, for ucOC; number of men was 200, 201, 199 and 203, respectively, for plasma phylloquinone, and 197, 200, 197 and 196, respectively, for ucOC; number of women was $228,231,229$ and 225, respectively, for plasma phylloquinone, and 224, 225, 223 and 224 , respectively, for ucOC $\ddagger$ Assessed using SAS PROC GLM.

$\S$ Geometric means adjusted for energy intake and plasma triacylglycerol.

|| Proportion of elevated ( $\geq 20 \%$ ) ucOC adjusted for energy intake.

and trans fatty acid intakes in the current study. A positive association was likewise observed between trans fatty acid intakes and detectable plasma dihydrophylloquinone concentrations. When assessed on the basis of individual food categories, there was a positive association between the primary dietary source of dihydrophylloquinone, bakery products and detectable plasma dihydrophylloquinone. Estimating trans fatty acid intake from products made with hydrogenated fat has been challenging in population-based studies because of the lack of a comprehensive database on trans fatty acid contents in foods and variable concentration of trans fatty acids in food items within a food category (Federal Register, 2003). Furthermore, the analysis of individual trans fatty acids in plasma or serum is elaborate and time consuming. Dihydrophylloquinone intake may better reflect dietary intake of hydrogenated fat than trans fatty acids because hydrogenation is the exclusive source of dihydrophylloquinone. One caveat to this conclusion is that dihydrophylloquinone is only formed during hydrogenation of phylloquinone-rich oils, which include soyabean, rapeseed, cottonseed and olive (Peterson et al. 2002). For those food supply systems that use hydrogenated plant oils that are not rich in phylloquinone, such as corn, peanut and safflower, dihydrophylloquinone would not be an appropriate marker of trans fatty acid intake.

A high serum \%ucOC is considered an indicator of poor vitamin K status (Sokoll \& Sadowski, 1996; Gundberg et al. 1998; Booth et al. 2001). Higher dihydrophylloquinone intakes tended to be associated with a higher proportion of elevated \%ucOC in the current study. These associations are consistent with prior metabolic data in humans (Booth et al. 2001). In contrast, plasma dihydrophylloquinone concentrations were not associated with \%ucOC, and at this point, the inconsistencies between the associations of \%ucOC with dietary versus plasma dihydrophylloquinone remain unresolved.

In summary, despite the limitations in the use of plasma dihydrophylloquinone, evident in the high proportion of the population that had non-detectable dihydrophylloquinone concentrations, plasma dihydrophylloquinone is positively associated with dihydrophylloquinone intake as measured using an FFQ. Furthermore, dihydrophylloquinone (both plasma and intake) are positively associated with trans fatty acid intake.

\section{Acknowledgements}

We gratefully acknowledge the Framingham Study participants and staff, Gail Rogers for statistical assistance and James W. Peterson for phylloquinone and dihydrophylloquinone analyses and Nathalie Weizmann for assistance with FFQ analyses. This material is based upon work supported by the US Department of Agriculture, under agreement No. 58-1950-4-401, the National Institute of Health (AG14759) and Academy of Finland (80232 and 79433).

\section{References}

Booth SL, Davidson KW, Lichtenstein AH \& Sadowski JA (1996a) Plasma concentrations of dihydro-vitamin K1 following dietary intake of a hydrogenated vitamin K1-rich vegetable oil. Lipids 31, 709-713.

Booth SL, Lichtenstein AH, O'Brien-Morse M, McKeoun NM, Wood RJ, Saltzman E \& Gundberg CM (2001) Effects of a hydrogenated form of vitamin $\mathrm{K}$ on bone formation and resorption. Am J Clin Nutr 74, $783-790$.

Booth SL, O'Brien-Morse ME, Dallal GE, Davidson KW \& Gundberg CM (1999a) Response of vitamin K status to different intakes and sources of phylloquinone-rich foods: comparison of younger and older adults. Am J Clin Nutr 70, 368-377.

Booth SL, Pennington JA \& Sadowski JA (1996b) Dihydro-vitamin K1: primary food sources and estimated dietary intakes in the American diet. Lipids 31, 715-720.

Booth SL \& Suttie JW (1998) Dietary intake and adequacy of vitamin K. J Nutr 128, 785-788.

Booth SL, Webb DR \& Peters JC (1999b) Assessment of phylloquinone and dihydrophylloquinone dietary intakes among a nationally 
representative sample of US consumers using 14-day food diaries. J Am Diet Assoc 99, 1072-1076.

Davidson KW, Booth SL, Dolnikowski GG \& Sadowski JA (1996) The conversion of phylloquinone to $2^{\prime}, 3^{\prime}$-dihydrophylloquinone during hydrogenation of vegetable oils. J Agric Food Chem 44, 980-983.

Davidson KW \& Sadowski JA (1997) Determination of vitamin K compounds in plasma or serum by high-performance liquid chromatography using postcolumn chemical reduction and fluorimetric detection. Methods Enzymol 282, 408-421.

Dumont JF, Peterson J, Haytowitz D \& Booth SL (2003) Phylloquinone and dihydrophylloquinone contents of mixed dishes, processed meats, soups and cheeses. J Food Compos Anal 16, 595-603.

Erkkilä AT, Lichtenstein AH, Dolnikowski GG, Grusak MA, Jalbert SM, Aquino KA, Petersen JW \& Booth SL (2004) Plasma transport of vitamin $\mathrm{K}$ in men using deuterium-labeled collard greens. Metabolism 53, 215-221.

Federal Register (2003) Food labeling: trans fatty acids in nutrition labeling, nutrient content claims, and health claims. Final rule. pp. 41433-41506. Food and Drug Administration, HHS.

Grundy SM (1998) Hypertriglyceridemia, atherogenic dyslipidemia, and the metabolic syndrome. Am J Cardiol 81, 18B-25B.

Gundberg CM, Nieman SD, Abrams S \& Rosen H (1998) Vitamin K status and bone health: an analysis of methods for determination of undercarboxylated osteocalcin. J Clin Endocrinol Metab 83, 3258-3266.

Kannel WB, Feinleib M, McNamara PM, Garrison RJ \& Castelli WP (1979) An investigation of coronary heart disease in families. The Framingham Offspring Study. Am J Epidemiol 110, 281-290.

Koivu T, Piironen V, Lampi A-M \& Mattila P (1999) Dihydrovitamin $K_{1}$ in oils and margarines. Food Chem 64, 411-414.

Lamon-Fava S, Sadowski JA, Davidson KW, O'Brien ME, McNamara JR \& Schaefer EJ (1998) Plasma lipoproteins as carriers of phylloquinone (vitamin $\mathrm{K}_{1}$ ) in humans. Am J Clin Nutr 67, 1226-1231.
Lichtenstein AH, Ausman LM, Jalbert SM \& Schaefer EJ (1999) Effects of different forms of dietary hydrogenated fats on serum lipoprotein cholesterol levels. N Engl J Med 340, 1933-1940.

McKeown NM, Jacques PF, Gundberg CM, Petersen JW, Tucker KL, Kiel DP, Wilson PW \& Booth SL (2002) Dietary and nondietary determinants of vitamin $\mathrm{K}$ biochemical measures in men and women. $J$ Nutr 132, 1329-1334.

McNamara JR \& Schaefer EJ (1987) Automated enzymatic standardized lipid analyses for plasma and lipoprotein fractions. Clin Chim Acta 166, $1-8$.

Mensink RP \& Katan MB (1990) Effect of dietary trans fatty acids on high-density and low-density lipoprotein cholesterol levels in healthy subjects. N Engl J Med 323, 439-445.

National Cholesterol Education Program (NCEP) Expert Panel (2002) Detection, evaluation, and treatment of high blood cholesterol in adults (Adult Treatment Panel III) final report. Circulation 106, 3143-3421.

Peterson JW, Muzzey KL, Haytowitz DB, Exler J, Lemar L \& Booth SL (2002) Phylloquinone (vitamin $\mathrm{K}_{1}$ ) and dihydrophylloquinone content of fats and oils. JAOCS 79, 641-646.

Rimm EB, Giovannucci EL, Stampfer MJ, Colditz GA, Libin LB \& Willett WC (1992) Reproducibility and validity of an expanded selfadministered semiquantitative food frequency questionnaire among male health professionals. Am J Epidemiol 135, 1114-1126.

Sokoll LJ \& Sadowski JA (1996) Comparison of biochemical indexes for assessing vitamin $\mathrm{K}$ nutritional status in a healthy adult population. Am $J$ Clin Nutr 63, 566-573.

Traber MG \& Jialal I (2000) Measurement of lipid-soluble vitamins further adjustment needed? Lancet 355, 2013-2014.

Willett WC, Sampson L, Stampfer MJ, Rosner B, Bain C, Witschi J, Hennekens CH \& Speizer FE (1985) Reproducibility and validity of a semiquantitative food frequency questionnaire. Am J Epidemiol 122, $51-65$. 